\title{
The Monetary Value of Informal Care: Obtaining Pure Time Valuations Using a Discrete Choice Experiment
}

\author{
Renske J. Hoefman ${ }^{1}$. Job van Exel ${ }^{1,2}$. Werner B. F. Brouwer ${ }^{1,2}$
}

Published online: 9 October 2018

(c) The Author(s) 2018

\begin{abstract}
Background Interventions in health care often not only have an effect on patients, but also on their informal caregivers. Caregiving can have a profound impact on the health and wellbeing of carers. Ignoring these spillovers in economic evaluations risks labelling interventions mistakenly as cost-effective, at the expense of informal caregivers.

Objective This paper investigates willingness-to-accept (WTA) values for an hour of informal care, corrected for positive and negative impacts of informal care, to facilitate the inclusion of informal care hours on the cost side of economic evaluations without double-counting spillover effects.

Methods A discrete choice experiment (DCE) was conducted among a representative sample of the adult population in the Netherlands $(n=552)$ in September 2011. An experimental design minimizing the D-error was used to construct choice sets with two unlabelled alternatives with the attributes 'hours caregiving', 'monetary compensation for caregiving' and seven impacts of caregiving. To operationalize the random utility model, we used a panel mixed multinomial logit (MMNL) parameter model. For calculation of WTA, we used both population-level parameters and individual-level parameters.

Results The mean WTA for an additional hour of informal care, corrected for positive and negative impacts of informal care, was $€ 14.57$. The signs of the coefficients were all in the expected directions.

Conclusions This study reports a preference-based monetary value for informal care, corrected for other impacts. This valuation facilitates the inclusion of informal care hours on the cost side in economic evaluations without double-counting any spillover effects included on the effects side.
\end{abstract}

Electronic supplementary material The online version of this article (https://doi.org/10.1007/s40273-018-0724-4) contains supplementary material, which is available to authorized users.

Job van Exel

vanexel@eshpm.eur.nl

1 Erasmus School of Health Policy and Management, Erasmus University Rotterdam, Bayle Building, Office J8-51, PO

Box 1738, 3000 DR Rotterdam, The Netherlands

2 Erasmus School of Economics, Erasmus University Rotterdam, Rotterdam, The Netherlands

\section{Key Points for Decision Makers}

Informal care is often not included in economic evaluations, while it could influence recommendations on the cost-effectiveness of health care interventions.

This study found a monetary value of informal care time of around $€ 14.50$ per hour, corrected for other impacts, among the public in the Netherlands.

Using this value, hours of informal care could easily be included at the cost side of economic evaluations without risk of double-counting spillover effects.

\section{Introduction}

A fundamental part of the care for frail elderly and ill and disabled people, such as cancer or dementia patients, is provided by their family members. The significance of this care 
is highlighted by the high percentages of people involved in caregiving in many Western countries, amounting to up to $30 \%$ of the population $[1,2]$. The vital role of informal caregivers is underlined by the ageing of populations and rising chronic disease rates in these countries, in combination with rationing policies restricting access to formal home care and support services.

Social relationships are the basis of informal caregiving, and hence, caregiving is often characterized by love and fulfilment. Nevertheless, many caregivers also feel burdened, especially when caregiving is intensive and demanding. In these situations, caregiving may lead to physical health problems, stress, depression, more medication use, and even increased mortality. The effect of patient health on others in their environment through the process of caregiving is a specific 'spillover effect', also known as the 'caregiving effect', and can be positive and negative. The health of patients can also influence the health of their loved ones directly, sometimes called the 'family effect' [3-6]. Therefore, altruistic preferences, i.e. that people care about the health and wellbeing of others, are relevant in health care in general and in the case of informal care specifically [4, 7-10]. Since caregivers often are family members of patients, the family effect and the caregiving effect will often occur simultaneously [11]. Note that spillover effects from caregiving may affect the health of caregivers, but also other aspects of their lives and wellbeing, such as their ability to perform paid work or to maintain social relationships [12-14].

The awareness that interventions may not only benefit patients but also others in their environment is crucial for well-informed decision making in health care, both at a clinical and a policy level. Recently, more attention for families in medical decisions has been advocated in leading clinical journals [15-17]. At a policy level, decision makers more and more are faced with challenging reimbursement decisions following on from the tension between scarce resources and rising demand for care. Economic evaluations comparing the costs and benefits of interventions are increasingly used to inform these decisions [18]. However, despite the recognition that patients are not isolated individuals, the costs and effects of illness and treatment on informal caregivers are often ignored in economic evaluations [19-21]. This may especially bias the results of economic evaluations in disease areas where informal care constitutes a large part of the total care provided or where the impact is large $[20,21]$. In any case, ignoring informal care is not in accordance with national guidelines for economic evaluations in some countries that advocate the societal perspective, which prescribes that all relevant costs and effects should be included in such evaluations, and thus also spillovers to informal care [4, 22-24]. But also in the context of a health care perspective, at least the health effects in informal caregivers (and broader family members) should be accounted for [25]. Attention for caregivers is also justified from an ethical point of view. Although including informal care may have distributional consequences, these can be explicitly considered when informal care is included. Ignoring the impact of caregiving results in ill-informed decisions and raises questions on distributional consequences of total health gains in society as well $[20,26]$.

One of the reasons for ignoring informal care in economic evaluations may be the lack of straightforward methods to include the effects of informal caregiving. Including the full impact of informal care in terms of wellbeing may indeed be difficult, but may be more feasible when restricting effects to the costs of hours of informal care provided and health effects [27]. When health effects in caregivers are included at the effect side of an economic evaluation, conventional health utility instruments could be applied and health utilities of caregivers could be added to the health utilities of patients at the effect side of a cost-utility analysis. However, including other aspects of caregiving beyond the healthrelated quality of life of caregivers is more challenging. At the cost side of economic evaluations, costs of informal care can easily be added to other costs related to an intervention. The challenging part here consists of the valuation of the time spent on caregiving. Given that informal care is a non-market and typically unpaid activity, the value of time needs to be determined. Different methods can be used for this purpose, including the opportunity costs or proxy good methods. These methods value the time spent on caregiving using wage rates of caregivers or professional substitutes [27-29]. However, these methods typically only focus on the value of alternative uses of time without considering the full impact of caregiving on caregivers, and hence do not necessarily reflect preferences of caregivers.

Some studies have attempted to derive a full valuation of caregiver time by applying for instance the wellbeing method, contingent valuation, or discrete choice experiments (DCEs) [14, 30-35]. Most of these studies focused on preference information of caregivers to derive a monetary value of time. To our knowledge, only one study has derived a value of time of both caregivers and non-caregivers using the contingent valuation method [32]. An important issue to consider in this context is that stated preference methods like WTP in theory produce a full valuation of informal care. Such a valuation would, in principle, include all spillover effects of providing informal care, including health effects. Obviously, this raises concerns about double-counting when other effects are also considered.

In this study, we will focus on the time costs of providing informal care. We derive valuations of time from a sample of the population in the Netherlands using the concept of willingness-to-accept (WTA) in a DCE. By correcting for other effects of informal care on caregivers, we aim to estimate a 'pure time cost' of an hour of informal 
care, which can be used in economic evaluations alongside other effects. Such a value could thus be used on the cost side of economic evaluations, if desired, in combination with health or wellbeing effects in caregivers.

\section{Methods}

\subsection{Data}

The data was collected with an online survey among a sample of the adult population in the Netherlands from a panel of an internet survey company in September 2011. Respondents were representative of the overall population in terms of age, gender and educational level. In cases where response time was under a third of the a priori estimated time to complete the survey (i.e. $12 / 3=4 \mathrm{~min}$ ), respondents were excluded by the survey company.

Which informal care situation do you prefer?

Informal care situation A
You provide 20 hours care per week
You have a lot of fulfilment from caregiving
You have a lot of relational problems with
the care recipient
You have some problems with your own
mental health
You have a lot of problems with combining
your daily activities with your care tasks
You have no financial problems with your
care tasks
You have some support with your care tasks
You have a lot of problems with your own
physical health
You receive 800 euros net per week from the
government for providing care

\subsection{Discrete Choice Experiment}

Preferences for caregiving were derived by eliciting stated preferences with a DCE. DCEs are often applied in research to investigate preferences of respondents, including in health care [36-38]. Before the start of the experiment, respondents received information about the aim of the study, the content of the survey, information on the topic of informal care, and an explanation of the attributes and levels. Respondents were instructed to imagine that they provided informal care for a person in their social network, and to keep this same person in mind during the whole experiment. An example of a choice task is presented in Fig. 1. Per choice question, two unlabeled informal care situations were presented, and respondents were asked which of two alternatives they preferred. The attributes were selected from previous choice experiments on preferences for caregiving describing the informal care situations by seven burden dimensions of a validated measure of quality of life of caregivers, the CarerQol instrument [27, 39-41]. This instrument includes the

Informal care situation B
You provide $\mathbf{4 0}$ hours care per week
You have a lot of fulfilment from caregiving
You have a lot of relational problems with
the care recipient
You have no problems with your own mental
health
You have no problems with combining your
daily activities with your care tasks
You have a lot of financial problems with
your care tasks
You have some support with your care tasks
You have some problems with your own
physical health
You receive 200 euros net per week from the
government for providing care

Fig. 1 Example of a choice task. Presented are descriptions of two informal care situations with nine characteristics. The descriptions differ as the levels of the characteristics are different. For example, no, some, or a lot of relational problems can be present in an informal care situation 
domains mental and physical health. To calculate WTA values for informal care, two attributes were added, these being (1) caregiving hours and (2) monetary compensation for caregiving.

The caregiving hours attribute was specified as the number of hours of caregiving per week, with six levels $(5,10$, $20,25,30$, and $40 \mathrm{~h}$ ). These levels are within the range of observed caregiving hours per week among a heterogeneous sample of informal caregivers in the Netherlands [42]. The seven CarerQol attributes were fulfilment from providing care, relational problems with the care recipient, mental health problems, problems with combining care with daily activities, financial problems with caregiving, support with caregiving, when needed, and physical health problems, all with three levels (no, some, and a lot). Colour-coding was applied to three levels of these seven burden attributes to enhance graphical presentation in the online survey, as previously done [40]. The attribute of 'monetary compensation for caregiving' consisted of a weekly net financial compensation for providing informal care from the government, with the levels $€ 0, € 200, € 400, € 500, € 600$, and $€ 800$. These levels were chosen to represent a realistic situation for respondents, while at the same time allowing a sufficiently wide range to observe differences in preferences. Previous studies have estimated monetary values of an hour of informal care to be around $€ 10$. Therefore, a fulltime work week spent on caregiving would amount to financial compensation of $€ 400$. The choice tasks were presented in random order to respondents.

We used an experimental design minimizing the D-error with two alternatives to construct 60 choice sets using Ngene (ChoiceMetrics 2011). These choice sets were randomly assigned over ten groups of respondents. Resembling the design of Hoefman et al.'s [40] work, the seven burden attributes were dummy coded and the six dummy attributes with the highest standard errors in that multinomial logit (MNL) model were treated as Bayesian priors with a normal distribution using 750 Halton draws, while optimizing for D-efficiency in the basic MNL model [43, 44]. The utility functions for the two alternatives also included one variable for the attribute for hours of caregiving, one variable for the attribute for monetary compensation, 36 interaction terms for all attribute combinations, and one constant term for the first alternative.

\subsection{Analyses}

The estimate of the WTA for an hour of informal care was derived in three steps. We first analysed the choice data with an MNL model in Nlogit. Second, as previous studies showed heterogeneity in the monetary valuation of care [31-33], we allowed for the presence of unobservable preference heterogeneity in the sampled population by applying a panel mixed multinomial logit (MMNL) model to derive the unconditional distribution of the random parameters in the model using 1000 Halton draws. Standard deviations of the (unconditional) parameter distribution were derived from the Cholesky matrix (see Table 1 for the Cholesky and correlation matrix of the panel MMNL model). The panel MMNL model also allowed for correlated parameters recognizing the correlation of unobserved effects among alternatives in a given choice situation. Different model specifications were tested with likelihood ratio tests. We tested (1) including an alternative specific constant, (2) the number of random parameters in the model, and (3) the linearity assumption of the monetary compensation attribute. Our final model included an alternative -specific constant. Moreover, we assumed all parameters (excluding the constant) to be continuous and random. In the analyses, higher scores on the two positive dimensions of the CarerQol (fulfilment from caring and receiving support) represented more fulfilment or support. Higher scores on the negative dimensions indicated fewer problems experienced by the caregiver. The attribute for monetary compensation was assumed to be log-normal, imposing the restriction of only positive values for this attribute. For the hours of caregiving and the seven CarerQol attributes, we did not use this restriction and hence used a normal distribution [33]. In the third step, we calculated WTA values for an hour of informal care using population-level and individual-level parameters.

\subsection{Willingness to Accept (WTA) for an Hour of Caregiving}

The panel MMNL model provides population-level estimates (unconditional distribution). We obtained the individual-level parameters from this unconditional distribution for random parameters using Nlogit [45]. Specifically, for each respondent we estimated the (conditional) mean and standard deviation for the 'monetary compensation' and 'hours of caregiving' parameters. Then, using these conditional parameter distributions for the 'hours of caregiving' and 'monetary compensation' attributes, we took 100 Halton draws out of the sample (from a normal distribution) per individual (55,200 draws in total) using bootstrap sampling in Microsoft Excel. We calculated the ratio of the 'hours of caregiving' and 'monetary compensation' individualspecific parameter estimates for each draw and obtained a mean WTA estimate per individual. Since we assumed a lognormal distribution which is characterized by an infinite tail in the positive direction, we deleted the $1 \%$ extreme values to calculate the mean (and median) WTA in the population using Microsoft Excel. 
Table 1 Estimates of Cholesky matrix (in lower, left diagonal elements of table) and correlation matrix [in upper (shaded) element of table] of panel MMNL model of Table 3

\begin{tabular}{|c|c|c|c|c|c|c|c|c|c|}
\hline & 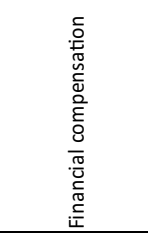 & 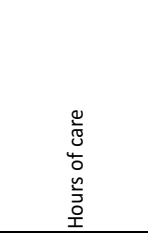 & 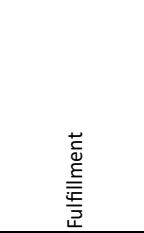 & 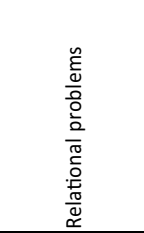 & 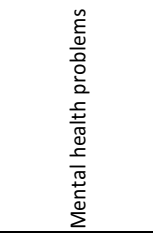 & 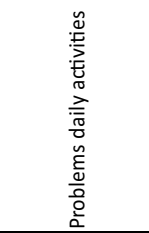 & 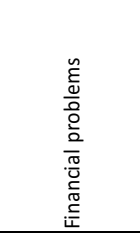 & $\begin{array}{l}5 \\
\text { 을 } \\
\frac{0}{3} \\
\tilde{n}\end{array}$ & 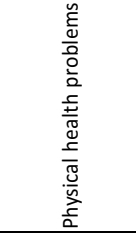 \\
\hline Financial compensation & $0.057(0.905)$ & -0.692 & -0.602 & 0.534 & 0.436 & 0.572 & 0.435 & 0.13 & 0.686 \\
\hline Hours of caregiving & $-1.025(0.304)$ & 0.049 (0.009) & 0.315 & 0.119 & -0.242 & -0.610 & -0.301 & -0.116 & -0.384 \\
\hline Fulfillment & $-0.598(0.370)$ & $0.257(0.186)$ & $0.775(0.162)$ & -0.259 & -0.257 & -0.257 & -0.858 & -0.440 & -0.354 \\
\hline Relational problems & $0.770(0.426)$ & $0.082(0.160)$ & $-0.215(0.162)$ & $0.650(0.136)$ & 0.167 & 0.149 & 0.095 & 0.184 & 0.522 \\
\hline Mental health problems & $0.183(0.490)$ & $-0.190(0.207)$ & $-0.148(0.228)$ & $0.114(0.185)$ & $0.737(0.179)$ & -0.126 & 0.252 & 0.243 & 0.409 \\
\hline Problems daily activities & $0.117(0.548)$ & $-0.40(0.142)$ & $-0.038(0.179)$ & $0.118(0.178)$ & $-0.188(0.172)$ & $0.379(0.185)$ & -0.140 & -0.492 & 0.321 \\
\hline Financial problems & $0.020(0.890)$ & $-0.142(0.132)$ & $-0.379(0.138)$ & $-0.060(0.171)$ & $0.023(0.197)$ & $-0.232(0.195)$ & $0.027(0.134)$ & 0.785 & 0.240 \\
\hline Support & $-0.277(0.700)$ & $-0.059(0.160)$ & $-0.215(0.180)$ & $0.035(0.205)$ & $0.067(0.201)$ & $-0.418(0.224)$ & $0.052(1.711)$ & $0.154(0.562)$ & 0.026 \\
\hline Physical health problems & $-2.253(0.949)$ & $-0.277(0.186)$ & $0.177(0.179)$ & $0.376(0.174)$ & $0.149(0.225)$ & $0.031(0.334)$ & $0.055(1.766)$ & $-0.374(0.845)$ & $0.325(1.065)$ \\
\hline
\end{tabular}

$M M N L$ mixed multinomial logit

${ }^{\text {a } H i g h e r ~ s c o r e s ~ r e p r e s e n t ~ m o r e ~ f i n a n c i a l ~ c o m p e n s a t i o n / m o r e ~ h o u r s ~}$

${ }^{\mathrm{b}}$ Higher scores represent more fulfilment from caring/more support

${ }^{\mathrm{c}}$ Higher scores represent fewer problems

\section{Results}

\subsection{Study Sample}

After exclusion of the respondents with a response time of less than 4 min on the survey (see Sect. 2), our final study sample consisted of 552 respondents. Table 2 presents background characteristics of the study sample and their familiarity with informal care. The mean age of respondents was 45 years, 53\% were female, and more than two thirds had a middle or high educational level. The large majority of respondents $(91.5 \%)$ indicated that they knew what informal care was before they participated in the study. Around one in four was a caregiver. More than two thirds of respondents did not have any experience with caregiving themselves.

\subsection{Preferences for Caregiving Situations}

Table 3 presents the relative weights of the attributes. In general, respondents preferred informal care situations where caregivers provided fewer hours of care. Receiving a monetary compensation from the government was positively associated with preferences for informal care situations. The utility for informal care situations was higher when these situations were characterized by more positive aspects of caregiving, i.e. more fulfilment from caregiving or more support with caregiving, or absence of negative aspects; this result was found for all five negative effects of the CarerQol$7 \mathrm{D}$, i.e. relational problems, mental health problems, problems with daily activities, financial problems, and physical health problems. The strongest influence was observed for mental and physical health problems.

\subsection{WTA for Informal Caregiving}

The WTA for caregiving was calculated based on the parameters shown in Table 3. First, using the individual-specific estimates of hours of caregiving and monetary compensation derived with bootstrap sampling, as described in the Sect. 2, we calculated the ratio of the 'hours of caregiving' and 'monetary compensation' individual-specific parameter estimates for each draw. Second, we calculated a mean WTA per individual by averaging the 100 WTA values per individual in our experiment (see Fig. 2). Third, we calculated the mean and median of these WTA values per individual to obtain mean and median WTA values in the population; the mean WTA value for an hour of caregiving, corrected for the other effects of caregiving included in the choice task, was $€ 14.57$, while the median WTA value for an hour of caregiving was $€ 4.80$.

\section{Discussion}

In this paper, we estimated the 'pure' value of time spent on caregiving among the adult population in the Netherlands using a DCE. Such an estimate facilitates the inclusion of the time costs of informal care in economic evaluations of health care interventions. In addition, we observed that preferences for informal caregiving were associated with the positive and negative effects caregivers may experience. In line with previous studies, we found that deriving fulfilment or having mental or physical health problems were especially important in this context [40, 41]. In general, respondents 
preferred informal care situations characterized by fewer hours a week and a higher financial compensation.

The overall WTA value per hour of informal care in our study was estimated to be around $€ 14.50$. This value needs some further discussion. First of all, previous studies estimated the value of informal care time to range approximately between $€ 1$ and $€ 30$ per hour (e.g. [14, 31-33, 35, 46]). Note that these values normally do not correct for the indirect influences of informal care on health and wellbeing domains, as done here. Hence, the fact that our current study found a value somewhat low in this range might well be explained

Table 2 Background characteristics and familiarity with informal care of the study sample, $n=552$

\begin{tabular}{ll}
\hline Characteristics & Mean (SD) or percentage \\
\hline Age, years & $45.5(15.8)$ (range 18-81) \\
Female & $53.3 \%$ \\
Educational level & \\
Low & $32.3 \%$ \\
Middle & $39.7 \%$ \\
High & $28.1 \%$ \\
Familiarity with informal care & \\
Knowledge of concept of informal care & $91.5 \%$ \\
Experiences with caregiving & \\
$\quad$ No & $67.6 \%$ \\
Caregiver (in the past) & $26.3 \%$ \\
Care recipient (in the past) & $6.0 \%$ \\
\hline
\end{tabular}

$S D$ standard deviation by the fact that other valuations may (implicitly) have had a broader scope. Here, we wanted to value only 'pure time costs' in order to allow the inclusion of health or wellbeing effects on the effect side of an economic evaluation, without the risk of double-counting.

Differences between studies in the estimated values of time could also be attributed to differences in the methodology applied as well as the framing of the hypothetical situation in the experiment. In that sense, it is good to emphasize some features of the current study. For example, in our DCE, we addressed the level of burden that caregivers would experience besides the number of hours and monetary compensation in the experiment, while in the DCE of Mentzakis et al. [33], the caregiving situation was described by the type of tasks performed, formal help received and monetary compensation obtained. Furthermore, differences could be due to the populations studied, either caregivers or non-caregivers (e.g. [47]), and their actual experience with caregiving tasks.

The value of an hour of informal care found in this study could be influenced by the absence of an opt-out alternative in the choice tasks. We intentionally 'forced' respondents to choose between two informal care situations because our aim was to investigate preferences for different caregiving situations and not for informal caregiving per se. Hence, we wanted to collect information on trade-offs within caregiving situations. Using an opt-out alternative would probably have resulted in consistently opting out by respondents who do not want to provide informal care. However, this may imply that our WTA estimate is an underestimate, because the utility for caregiving situations may be overestimated.
Table 3 Results of MNL and panel MMNL model of preferences for caregiving, $n=552$

\begin{tabular}{|c|c|c|c|c|c|c|}
\hline & \multicolumn{3}{|c|}{ MNL model } & \multicolumn{3}{|c|}{$\begin{array}{l}\text { MMNL unconditional param- } \\
\text { eter distribution }\end{array}$} \\
\hline & Coef. & SE & $P$ value & Coef. & SD & $P$ value \\
\hline $\begin{array}{l}\text { Financial compensation per week from } \\
\text { government in euros }^{\mathrm{a}}\end{array}$ & 0.001 & 0.000 & 0.000 & 0.001 & 1.481 & 0.000 \\
\hline Hours of caregiving per week ${ }^{\mathrm{a}}$ & -0.019 & 0.003 & 0.000 & -0.034 & 0.049 & 0.000 \\
\hline Fulfilment from caregiving ${ }^{\mathrm{b}}$ & 0.559 & 0.039 & 0.000 & 1.001 & 0.816 & 0.000 \\
\hline Relational problems of caregiver $^{c}$ & 0.373 & 0.035 & 0.000 & 0.703 & 0.689 & 0.000 \\
\hline Mental health problems of caregiver ${ }^{\mathrm{c}}$ & 0.524 & 0.046 & 0.000 & 0.959 & 0.783 & 0.000 \\
\hline Problems with daily activities of caregiver ${ }^{c}$ & 0.271 & 0.039 & 0.000 & 0.509 & 0.557 & 0.000 \\
\hline Financial problems of caregiver ${ }^{c}$ & 0.334 & 0.037 & 0.000 & 0.682 & 0.471 & 0.000 \\
\hline Support with caregiving ${ }^{\mathrm{b}}$ & 0.165 & 0.040 & 0.000 & 0.344 & $0.506^{*}$ & 0.001 \\
\hline Physical health problems of caregiver ${ }^{\mathrm{c}}$ & 0.508 & 0.041 & 0.000 & 0.922 & 0.722 & 0.000 \\
\hline Constant of alternative $\mathrm{A}$ & 0.078 & 0.040 & 0.050 & 0.120 & - & 0.022 \\
\hline
\end{tabular}

$C I$ confidence interval, Coef. coefficient, $M M N L$ mixed multinomial logit, $M N L$ multinomial logit, $S D$ standard deviation, $S E$ standard error

*SD of parameter distribution not statistically significant at $90 \%$ CI

${ }^{a}$ Higher scores represent more financial compensation/more hours

${ }^{\mathrm{b}}$ Higher scores represent more fulfilment from caring/more support

${ }^{c}$ Higher scores represent fewer problems 
This means that respondents chose alternatives with a level of monetary compensation which is not high enough for them to provide informal care in real life.

In addition, modelling choices may have influenced the results of our study. As an illustration, estimates of WTA values were somewhat higher when using a model with all fixed parameters (MNL model) or a model with the monetary compensation attribute as a fixed parameter (while assuming a distribution for the other attributes): respectively, values of $€ 19$ (ratio of $\beta$ hours of caregiving and $-\beta$ monetary compensation of the coefficients of the MNL model presented in Table 3, second column) and $€ 17$ for an hour of caregiving [derived by the same procedure as explained in the Sect. 2 using individual-specific estimates of $\beta$ hours of caregiving divided by the fixed parameter estimate for monetary compensation (see the Appendix Table 4) for each draw in the Bootstrap sampling].

In this study, we chose to model preference heterogeneity as a continuous function, as this allowed us to estimate individual-specific parameters that could be averaged-using draws from the conditional parameter distribution-to calculate an average monetary compensation for an hour of informal care for the whole population that could be applied in the context of economic evaluations. Doing so, we applied the same modelling procedure as previously used in DCEs on preferences for informal caregiving [41], of which this DCE is an extension by adding information on the hours of caregiving and monetary compensation to the choice tasks (in order to calculate WTA). As stressed in the literature, selecting appropriate models for choice data is sometimes challenging, especially given the lack of theoretical foundation for such selections, for instance, the type of distribution of the continuous preference heterogeneity [37, 48].
For example, we chose a lognormal distribution for the cost attribute. While this assumption is more realistic and in line with economic theory than applying a normal distribution for a cost attribute, this may impose problems with calculating ratios of attributes for WTA calculation given the infinite tail in the positive direction of the cost attribute [49]. To address this, we deleted the $1 \%$ extreme values of obtained WTA values.

Another noteworthy feature of our study is that we used a sample of the general population to answer this value of time question, in contrast to most previous studies. The responses of our sample indicated that a relatively small group of $14 \%$ of respondents found it hard to imagine being an informal caregiver. This modest percentage supports the feasibility of studying the value of time of caregiving in a stated preference study among the general population. The results of Garrido-García et al. [32] also support the feasibility of deriving a value of caregiving time among non-caregivers. Still, it needs emphasis that we used this relatively inexperienced sample to estimate the value of an hour of informal care. Furthermore, it should be noted that care situations in our experiment were described in terms of experienced burden from caregiving, e.g. whether caregivers experienced physical or mental health problems. This raises the question as to whether this type of information concerning experienced problems could be used in these types of experiments. Furthermore, the attribute fulfilment deserves some discussion. Fulfilment from providing care tasks is commonly considered to be an important (positive) domain of caregiver quality of life. It is, for instance, included in the care-related quality-of-life measure Caregiver Experience Scale (CES), and similar positive items are part of the burden measure Caregiver Strain

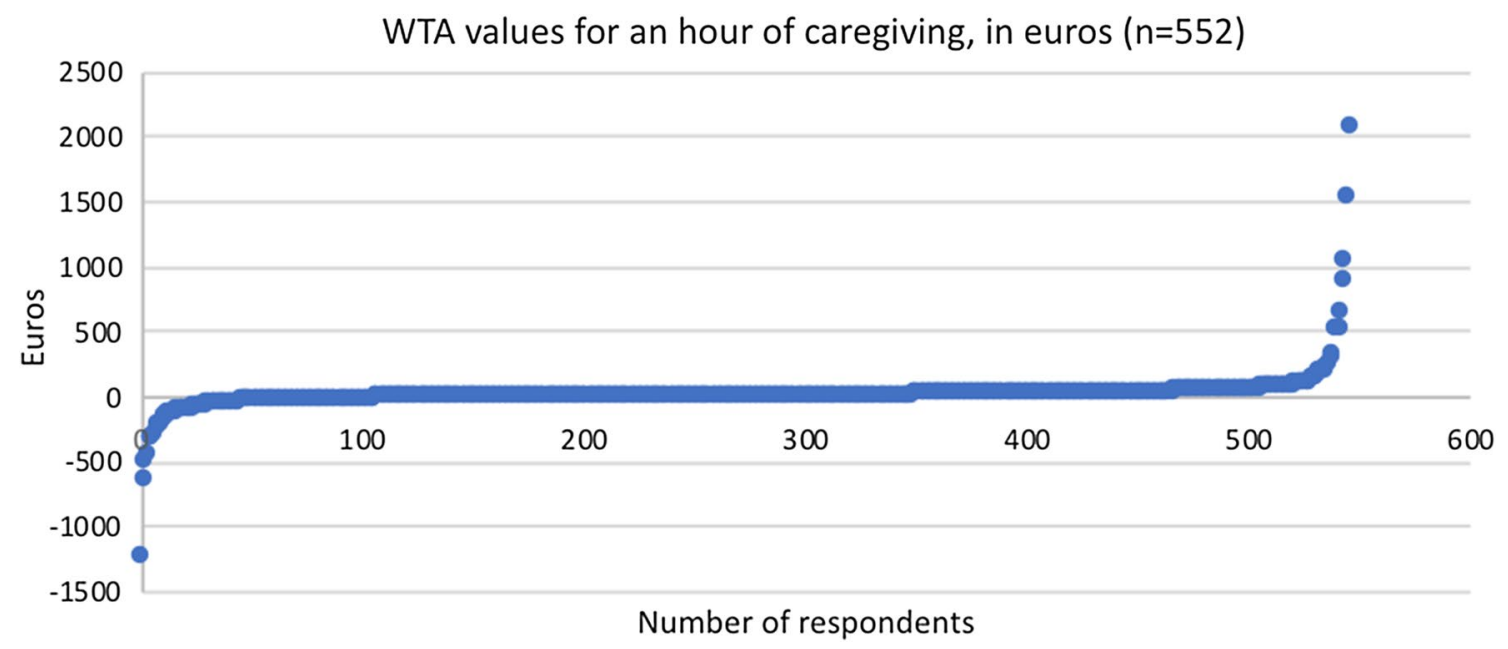

Fig. 2 Willingness-to-accept (WTA) values for an hour of caregiving, in euros $(n=552)$ 
Index $+[50]$. One might also interpret fulfilment as some kind of overall evaluation of the caregiving situation, which would make it troublesome to include it as an attribute in a DCE aiming to arrive at such an overall evaluation. However, in the context of the CarerQol, fulfilment is regarded as one of the domains that jointly with the other six domains results in an overall valuation of a caregiving situation. A caregiver may derive no fulfilment from providing care but still judge the overall situation favourably if the scores on the other domains are good. Similarly, a caregiver can derive a lot of fulfilment from providing care, in spite of low scores on the other domains. In the development of the CarerQol, the current DCE, or in previous DCEs conducted to obtain utility scores for the CarerQol, we found no indication that respondents interpreted the attribute fulfilment as an overall indication of utility. The choice of an appropriate source of information, especially if this would affect the results, remains something to be considered, like in the case of health state valuations [51]. Nevertheless, repeating this study in a sample of caregivers would be an interesting option for future research.

Finally, we would like to emphasize that the value of caregiving time reported here was obtained in the Netherlands in 2011. Further studies should investigate whether this value also correctly reflects the 'pure' value of time spent on caregiving in other countries than the Netherlands, and whether this value has changed over time. In addition, we estimated an average value per hour of informal care provided. Previous studies indicated there may be considerable heterogeneity in preferences for type of care task performed [33, 34, 52, 53]. Future research could therefore focus on the valuation of time performing different informal care tasks. Furthermore, preference heterogeneity can be expected between groups of respondents, such as those with a low or high income, and males or females. The objective of this paper was to obtain one value of time of caregiving reflecting preferences of the general public in the Netherlands for use in economic evaluations. Notwithstanding this, it would be interesting in future studies to investigate differences in preferences for caregiving situations between respondents, for example, using latent-class analysis.

The WTA value per hour of caregiving derived in this study could be used on the cost side of economic evaluations. The 'pure' time costs of informal care can be computed by multiplying the hours of caregiving with the estimate of around $€ 14.50$ per hour, and then add this to the other cost components in the economic evaluation. Given that this value was corrected for broader health and wellbeing impacts of informal care, this estimate also allows the inclusion of these broader spillover effects of informal caregiving on the effect side of the economic evaluation without the risk of double-counting. An advantage of this strategy is that informal care can be included in diverse types of cost-effectiveness studies, including cost-utility analyses. Furthermore, informal care costs can be included in reference cases, to present the cost-effectiveness results with and without informal care [23].

\section{Conclusions}

This paper provides a monetary value of an hour of informal caregiving based on preferences of the adult population in the Netherlands to facilitate the inclusion of the time costs of informal care in economic evaluations. Researchers can use this value to compute these time costs and add them to the other cost components in economic evaluations. Given the way these values were derived, this still allows the inclusion of other spillover effects in the effects side of economic evaluations. Outcomes in caregivers can thus be included in cost-effectiveness studies informing policy makers and, hence, stimulating health and welfare decision making in health care.

Author contributions RJH, JvE and WBFB designed the study. RJH coordinated the data collection, performed the data analyses, and wrote the draft manuscript. JvE and WBFB provided feedback on the draft manuscript. RJH, JvE and WBFB finalized the manuscript.

Funding No funding was received for this study.

Data availability statement The survey, design, software code for analysis, and the dataset are provided in the electronic supplementary material.

\section{Compliance with Ethical Standards}

Conflict of interest RJH has no conflicts of interest. JvE has no conflicts of interest. WBFB has no conflicts of interest.

Open Access This article is distributed under the terms of the Creative Commons Attribution-NonCommercial 4.0 International License (http://creativecommons.org/licenses/by-nc/4.0/), which permits any noncommercial use, distribution, and reproduction in any medium, provided you give appropriate credit to the original author(s) and the source, provide a link to the Creative Commons license, and indicate if changes were made.

\section{Appendix}

See Table 4. 
Table 4 Results of panel MMNL model of preferences for caregiving with monetary compensation as a fixed parameter, $n=552$

\begin{tabular}{lrll}
\hline & \multicolumn{3}{l}{$\begin{array}{l}\text { MMNL unconditional } \\
\text { parameter distribution }\end{array}$} \\
\cline { 2 - 4 } & Coef. & SD & $P$ value \\
\hline $\begin{array}{l}\text { Financial compensation per week from } \\
\quad \text { government, in euros }\end{array}$ & 0.002 & $0.000^{*}$ & 0.000 \\
Hours of caregiving per week & -0.027 & 0.044 & 0.000 \\
Fulfilment from caregiving & 0.891 & 0.706 & 0.000 \\
Relational problems of caregiver & 0.621 & 0.724 & 0.000 \\
Mental health problems of caregiver & 0.796 & 0.627 & 0.000 \\
Problems with daily activities of car- & 0.421 & 0.497 & 0.000 \\
$\quad$ egiver & & & \\
Financial problems of caregiver & 0.532 & 0.402 & 0.000 \\
Support with caregiving & 0.247 & 0.459 & 0.002 \\
Physical health problems of caregiver & 0.758 & 0.529 & 0.000 \\
Constant of alternative A & 0.121 & $0.059^{*}$ & 0.049 \\
\hline
\end{tabular}

Coef. coefficient, $M M N L$ mixed multinomial logit, $S D$ standard deviation, $S E$ standard error

*SE for fixed parameter

\section{References}

1. Colombo F, Llena-Nozal A, Mercier J, Tjadens F. Help wanted? Providing and paying for long-term care. OECD Health Policy Studies, OECD Publishing; 2011. https://doi.org/10.1787/97892 64097759-en.

2. de Klerk M, de Boer A, Plaisier I, Schyns P, Kooiker S. Informal care: who performs what? [Informele hulp: wie doet er wat?]. Netherlands Inst Soc Res. The Hague. SCP report 2015-35. 2015.

3. Bobinac A, Van Exel NJ, Rutten FF, Brouwer WB. Caring for and caring about: disentangling the caregiver effect and the family effect. J Health Econ. 2010(4);29:549-56.

4. Brouwer WBF. Too important to ignore. Pharmacoeconomics. 2006;24(1):39-41.

5. Wittenberg E, Saada A, Prosser LA. How illness affects family members: a qualitative interview survey. Patient Patient Centered Outcomes Res. 2013;6(4):257-68.

6. Wittenberg E, Ritter GA, Prosser LA. Evidence of spillover of illness among household members EQ-5D scores from a US sample. Med Decis Mak. 2013;33(2):235-43.

7. Al-Janabi H, Van Exel J, Brouwer W, Coast J. A framework for including family health spillovers in economic evaluation. Med Decis Mak. 2016;36(2):176-86.

8. Culyer AJ. The nature of the commodity 'health care' and its efficient allocation. Oxf Econ Pap. 1971;23(2):189-211.

9. Hurley J, Mentzakis E. Health-related externalities: evidence from a choice experiment. J Health Econ. 2013;32(4):671-81.

10. Jacobsson F, Carstensen J, Borgquist L. Caring externalities in health economic evaluation: how are they related to severity of illness? Health Policy. 2005;73(2):172-82.

11. Bobinac A, van Exel N, Rutten FFH, Brouwer WBF. Health effects in significant others. Med Decis Mak. 2011;31(2):292-8.

12. Chappell NL, Reid RC. Burden and well-being among caregivers: examining the distinction. Gerontologist. 2002;42(6):772-80.

13. Payakachat N, Tilford JM, Brouwer WBF, van Exel NJ, Grosse SD. Measuring health and well-being effects in family caregivers of children with craniofacial malformations. Qual Life Res. 2011;20(9):1485-95.

14. Van den Berg B, Fiebig DG, Hall J. Well-being losses due to caregiving. J Health Econ. 2014;35:123-31.

15. Al-Janabi H, Nicholls J, Oyebode JR. The need to "carer proof" healthcare decisions. BMJ. 2016;352:i1651.

16. Adelman RD, Tmanova LL, Delgado D, Dion S, Lachs MS. Caregiver burden: a clinical review. J Am Med Assoc. 2014;311(10):1052-60.

17. Wittenberg E, Prosser LA. Health as a family affair. N Engl J Med. 2016;374(19):1804-6.

18. Brouselle A, Lessard C. Economic evaluation to inform health care decision-making: promise, pitfalls and a proposal for an alternative path. Soc Sci Med. 2011;72(6):832-9.

19. Basu A, Meltzer D. Implications of spillover effects within the family for medical cost-effectiveness analysis. J Health Econ. 2005;24(4):751-73.

20. Goodrich K, Kaambwa B, Al-Janabi H. The inclusion of informal care in applied economic evaluation: a review. Value Health. 2012;15(6):975-81.

21. Krol M, Papenburg J, van Exel J. Does including informal care in economic evaluations matter? A systematic review of inclusion and impact of informal care in cost-effectiveness studies. Pharmacoeconomics. 2015;33(2):123-35.

22. Hakkaart-van Roijen L, Van der Linden N, Bouwmans C, Kanters T, Tan SS. Kostenhandleiding. Methodol van kostenonderzoek en Ref voor Econ Eval gezondheidszorg. Opdr van Zorginstituut Ned versie. Guideline for conducting economic evaluations in healthcare [in Dutch: Richtlijn voor het uitvoeren vaneconomische evaluaties in de gezondheidszorg]. 2015.

23. Sanders GD, Neumann PJ, Basu A, Brock DW, Feeny D, Krahn M, et al. Recommendations for conduct, methodological practices, and reporting of cost-effectiveness analyses: second panel on cost-effectiveness in health and medicine. Jama. 2016;316(10):1093-103.

24. Van Exel J, Bobinac A, Koopmanschap M, Brouwer W. The invisible hands made visible: recognizing the value of informal care in healthcare decision-making. Expert Rev Pharmacoecon Outcomes Res. 2008;8(6):557-61.

25. Al-Janabi H, Van Exel J, Brouwer W, Trotter C, Glennie L, Hannigan $\mathrm{L}$, et al. Measuring health spillovers for economic evaluation: a case study in meningitis. Health Econ. 2016;25(12):1529-44.

26. Al-Janabi H, Manca A, Coast J. Predicting carer health effects for use in economic evaluation. PLoS One. 2017;12(9):e0184886.

27. Hoefman RJ, van Exel J, Brouwer W. How to include informal care in economic evaluations. Pharmacoeconomics. 2013;31(12):1105-19.

28. Koopmanschap MA, Van Exel JN, Van den Berg B, Brouwer WB. An overview of methods and applications to value informal care in economic evaluations of healthcare. Pharmacoeconomics. 2008;26(4):269-80.

29. Oliva-Moreno J, Trapero-Bertran M, Peña-Longobardo LM, del Pozo-Rubio R. The valuation of informal care in costof-illness studies: a systematic review. Pharmacoeconomics. 2017;35(3):331-45.

30. Van den Berg B, Al M, Van Exel J, Koopmanschap M, Brouwer W. Economic valuation of informal care: conjoint analysis applied in a heterogeneous population of informal caregivers. Value Health. 2008;11(7):1041-50.

31. Van den Berg B, Brouwer W, Van Exel J, Koopmanschap M. Economic valuation of informal care: the contingent valuation method applied to informal caregiving. Health Econ. 2005;14(2):169-83.

32. Garrido-García S, Sánchez-Martínez FI, Abellán-Perpiñán JM, Van Exel J. Monetary valuation of informal care based on carers' and noncarers' preferences. Value Health. 2015;18(6):832-40. 
33. Mentzakis E, Ryan M, McNamee P. Using discrete choice experiments to value informal care tasks: exploring preference heterogeneity. Health Econ. 2011;20(8):930-44.

34. Schneider U, Kleindienst J. Monetising the provision of informal long-term care by elderly people: estimates for European outof-home caregivers based on the well-being valuation method. Health Soc Care Community. 2016;24(5):e81-91.

35. Gervès-Pinquié $\mathrm{C}$, Bellanger MM, Ankri J. Willingness to pay for informal care in France: the value of funding support interventions for caregivers. Health Econ Rev. 2014;4(1):34.

36. Bekker-grob EWDE, Ryan M, Gerard K. Discrete choice experiments in health economics: a review of the literature. Health Econ. 2012;21(2):145-72.

37. Sagebiel J. Preference heterogeneity in energy discrete choice experiments: a review on methods for model selection. Renew Sustain Energy Rev. 2017;69:804-11.

38. Clark MD, Determann D, Petrou S, Moro D, de Bekker-Grob EW. Discrete choice experiments in health economics: a review of the literature. Pharmacoeconomics. 2014;32(9):883-902.

39. Brouwer WB, Van Exel NJ, Van Gorp B, Redekop WK. The CarerQol instrument: a new instrument to measure care-related quality of life of informal caregivers for use in economic evaluations. Qual life Res. 2006;15(6):1005-21.

40. Hoefman RJ, Van Exel NJA, Rose JM, Lawerman-van de Wetering EJ, Brouwer WBF. A discrete choice experiment to obtain a tariff for valuing informal care situations measured with the CarerQol instrument. Med Decis Mak. 2014;34(1):84-96.

41. Hoefman RJ, van Exel J, Brouwer WBF. Measuring care-related quality of life of caregivers for use in economic evaluations: CarerQol tariffs for Australia, Germany, Sweden, UK, and US. Pharmacoeconomics. 2017;35(4):469-78.

42. Hoefman RJ, van Exel J, Brouwer WB. Measuring the impact of caregiving on informal carers: a construct validation study of the CarerQol instrument. Health Qual Life Outcomes. 2013;11(1):173-86.
43. Bliemer MCJ, Rose JM. Experimental design influences on stated choice outputs: an empirical study in air travel choice. Transp Res Part A Policy Pract. 2011;45(1):63-79.

44. Rose JM, Bliemer MCJ. Constructing efficient stated choice experimental designs. Transp Rev. 2009;29(5):587-617.

45. Greene W. Nlogit. Version 5.0. Reference guide, Econometric Software INC. 1st ed. US: Plainview; 2007.

46. Goossens LMA, Utens CMA, Smeenk FWJM, Donkers B, Van Schayck OCP, Rutten-Van Molken MPMH. Should I stay or should I go home? A latent class analysis of a discrete choice experiment on hospital-at-home. Value Health. 2014;17(5):588-96.

47. Malhotra C, Farooqui MA, Kanesvaran R, Bilger M, Finkelstein E. Comparison of preferences for end-of-life care among patients with advanced cancer and their caregivers: a discrete choice experiment. Palliat Med. 2015;29(9):842-50.

48. Hensher DA, Greene WH. The mixed logit model: the state of practice. Transportation. 2003;30(2):133-76.

49. Daly A, Hess S, Train K. Assuring finite moments for willingness to pay in random coefficient models. Transportation. 2012;39(1):19-31.

50. Al-Janabi H, Frew E, Brouwer WBF, Rappange D, Van Exel NJA. The inclusion of positive aspects of caring in the Caregiver Strain Index: tests of feasibility and validity. Int J Nurs Stud. 2010;47(8):984-93.

51. Versteegh MM, Brouwer WBF. Patient and general public preferences for health states: a call to reconsider current guidelines. Soc Sci Med. 2016;165:66-74.

52. De Meijer C, Brouwer W, Koopmanschap M, Van den Berg B, Van Exel J. The value of informal care-a further investigation of the feasibility of contingent valuation in informal caregivers. Health Econ. 2010;19(7):755-71.

53. Van den Berg B, Bleichrodt H, Eeckhoudt L. The economic value of informal care: a study of informal caregivers' and patients' willingness to pay and willingness to accept for informal care. Health Econ. 2005;14(4):363-76. 